\title{
DISTRIBUTION OF NEUROPEPTIDES IN NASAL AND NASOPHARYNGEAL MUCOSA IN PATIENTS WITH THE POST NASAL DRIP SYNDROME
}

\author{
Gunta Sumeraga ${ }^{1}$, Mara Pilmane ${ }^{2}$ \\ ${ }^{1}$ Pauls Stradins Clinical University Hospital, \\ Department of Otorhinolaryngology, Riga, Latvia \\ ${ }^{2}$ Institute of Anatomy and Anthropology, Riga Stradins University, Riga, Latvia
}

\begin{abstract}
OBJECTIVE The post nasal drip is a very common symptom of sinusitis, allergic rhinosinusopathy, the gastroesophageal reflux disease, but there are some patients, who have a post nasal drip, sensation of a foreign body in the nasopharynx and a non-specific irritant cough with no others symptoms or signs of sinus inflammations or allergy. In the posterior rhinoscopy mucus discharge is seen. Ethiology and pathogenesis of this syndrome evolution are still unclear. The aim of the study was to identify the neuropeptide appearance and distribution in nasal and nasopharyngeal mucosa in the patients with the isolated post nasal drip syndrome and the control group, for the comparison of the data.

MATERIALS AND METHODS. We investigated the biopsies of nasal and nasopharyngeal mucosa from 11 adult patients, who had the isolated post nasal drip syndrome and from 2 control group patients without post nasal drip, by conventional light microscopy and immunohistological techniques for the protein gene product 9.5 (PGP), Neuropeptide Y (NPY), serotonin, Substance P (SP), vasoactive intestinal peptide (VIP), Calcitonin gene related peptide (CGRP) and Chromogranin A (CgA).

RESULTS. The conventional light microscopy showed a very thick basal membrane, the sclerosis of small blood vessels, the hyperplasia of mucosal glands- the neurogenic inflammation, mostly in nasopharyngeal mucosa. The abundance of PGP-containing nerve fibres was found around glands, sclerotic arterioles in almost all the cases. The main neuropeptides that were found in the mucosa of the patients were VIP, NPY, CgA, mostly in the nasopharyngeal mucosa. There were diffe-
\end{abstract}


rences in PGP-containing structures and the selective neuropeptide (NPY, CgA, and VIP) distribution in nasal and nasopharyngeal mucosa in comparison with the control group.

CONCLUSIONS. The main histological changes in the patients with the isolated post nasal drip syndrome are a thickened basal membrane, the hyperplasia of basal cells, pronounced hyperplasia of mucosal glands, the sclerosis of small arterioles. The main neuropeptides that are found in nasal and nasopharyngeal mucosa samples are PGP 9.5, vasoactive intestinal peptide, neuropeptide $\mathrm{Y}$ and chromogranin in the post nasal drip syndrome group. There is an imbalance of common neuropeptide-containing innervations, mainly sympathic nerves, the precursors of neuropeptides- chromogranins in the nasopharyngeal mucosa of the patients with the post nasal drip syndrome. SP and CGRP are not the most characteristic neuropeptides in the case of the post nasal drip syndrome pathogenesis.

Key words: neuropeptide, neurogenic inflammation, nasal mucosa.

\section{INTRODUCTION}

The post nasal drip is usually a symptom of acute or chronic rhinosinusitis, allergic rhinosinusitis, gastroesophageal reflux disease. There is a group of patients, who have no other symptoms of sinus inflammation, allergy or reflux, but still they have mucus discharge in nasoparynx, the sensation of a foreign body in the pharynx and irritant cough, the so-called post nasal drip syndrome (23).

The nasal mucosa is richly innervated by sensory, sympathetic and parasympathetic nerve fibres which secrete, when they become activated, a variety of transmitter molecules. The source of neuropeptides may be the nerve cells and the neurons associated cells, like glyocytes and fibroblasts. $\mathrm{C}$ and $\mathrm{A} \delta$ fiber stimulation causes the release of neuropeptides. The release of neuropeptides by sensory nerve endings produces vasodilatation and increased vascular permeability, the phenomena primarily described in the rodents that have been collectively termed neurogenic inflammation $(4,16,22)$.

The substance P (SP), serotonin, the calcitonin gene related peptide (CGRP), the gastrine releasing peptide, the neuropeptide Y (NPY), the vasoactive intestinal peptide (VIP), chromogranin are found in nasal mucosa (4). These neuropeptides cause vasodilatation and upper airways 
oedema, characterized by nasal obstruction, and bronchial constrictions, increased mucus formation, the leukocyte recruitment and differentiation and the activation of various immune cells, including lymphocytes, eosinophils, mast cells, and macrophages $(23,24)$.

In the case of chronic upper and lower respiratory tract illnesses, respiratory mucosa shows an increased quantity of pro-inflammatory sensory neuropeptides. Neuropeptide concentrations correlate with the patients' symptom intensity (16).

The concentration of neuropeptides in the bronchial and nasal secretions is relatively low for asymptomatic patients, but it is highly elevated in the patients with allergic respiratory diseases, including nasal polyps $(4,7)$. Since neuroendocrine cells in the airway mucosa are discovered, increasing interest is attached to the yet unexplored neurogenic inflammation in the case of chronic airway inflammation, especially if it is characterized by increased airway reactivity.

The aim of the study was to identify neuropeptide appearance and distribution in nasal and nasopharyngeal mucosa in the patients with the isolated post nasal drip syndrome and the patients without the post nasal drip and to compare the data.

\section{MATERIAL AND METHODS}

Inferior nasal turbinate and nasopharyngeal mucosa specimens were obtained from 11 adult patients (age 18-58) with the isolated post nasal drip syndrome and from 2 control group patients (age 28-36).

All the patients were examinated by an otolaryngologist to exclude rhinosinusopathy (the normal pneumatisation of all the sinuses at the CT scan), allergy (no changes in skin prick tests, IgE range (total and specific)) and gastroesophageal reflux disease (clinically and by performing endofibrogastroscopy). All the patients have had symptoms of the post nasal drip for more than 6 months and had no reaction to common therapy: topical steroid (fluticasone), antihistamine (loratidin), anti-reflux therapy (diet and omeprasole).

The control group was formed from 2 voluntary patients, who where undergoing rhinoseptoplasty, these patients where without any symptoms of the post nasal drip.

Tissue pieces $\left(1-2 \mathrm{~mm}^{2}\right)$ from the middle part of the lower nasal turbinate and the middle part of the nasopharyngeal arch area were taken 
under control of the endoscope, under local anaesthesia (submucosal administration of $1-2 \mathrm{ml}$ of $1 \%$ lidocaine solution) or general anaesthesia (control group).

For immediate fixation previously prepared saturated picric acid solution (formaldehyde $2 \%, 0.2 \%$ o Picric acid, $1 \mathrm{M}$ phosphate buffered $(\mathrm{pH}$ 7.2) was used. Tissue slices were stained with haematoxylin and eosin, and by the use of immunohistological technique (Hsu et al 1981) for the protein gene product 9.5 (PGP), substance P (SP), Neuropeptide Y (NPY), vasoactive intestinal peptide (VIP), serotonin, calcitonin gene related peptide (CGRP) and chromogranin A (CgA) (Table 1).

The samples were examined under the Leica microscope. The results of immunohistochemistry were listed by the semi-quantitative counting method (Tobin et al., 1990; Pilmane, 1997) (Table 2).

Table 1. The data of the antibodies applied in immunohistochemistry

\begin{tabular}{|l|l|c|l|c|}
\hline & $\begin{array}{l}\text { Obtained } \\
\text { from }\end{array}$ & $\begin{array}{c}\text { Working } \\
\text { dilution }\end{array}$ & Manufacturer & Code \\
\hline PGP & rabbit & $1: 600$ & DAKO (Denmark) & Z5116 \\
\hline Serotonin & mouse & $1: 10$ & DAKO (Denmark) & M758 \\
\hline NPY & rabbit & $1: 10$ & DAKO (Denmark) & B48-100 \\
\hline VIP & rabbit & $1: 400$ & abcam (UK) & Ab22736 \\
\hline SP & mouse & $1: 1000$ & abcam (UK) & Ab14184 \\
\hline CGRP & rabbit & $1: 20$ & Quartet (Germany) & 281328 \\
\hline CgA & rabbit & $1: 400$ & DAKO (Denmark) & A0430 \\
\hline
\end{tabular}


Table 2. The semi-quantitative analysis of the immunohistochemically determined structures

\begin{tabular}{|l|l|}
\hline $\begin{array}{l}\text { Applied } \\
\text { markings }\end{array}$ & Semiquantitive explantation \\
\hline- & No positive structure seen in the visual field \\
\hline $0 /+$ & Rare positive structures seen in the visual field \\
\hline+ & Few positive structures seen in the visual field \\
\hline$+/++$ & $\begin{array}{l}\text { Few to a moderate number of positive structures seen in the } \\
\text { visual field }\end{array}$ \\
\hline++ & $\begin{array}{l}\text { A moderate number of positive structures seen in the visual } \\
\text { field }\end{array}$ \\
\hline$++/+++$ & $\begin{array}{l}\text { Moderate to numerous positive structures seen in the visual } \\
\text { field }\end{array}$ \\
\hline+++ & Numerous positive structures seen in the visual field \\
\hline$+++/++++$ & The abundance of positive structures in the visual field \\
\hline
\end{tabular}

\section{RESULTS}

Nasal mucosa demonstrated the pronounced partially patchy thickened basal membrane. Epithelium showed basal cell hyperplasia and intraepithelial infiltration with lymphocytes, marked hyperplasia of glandulocytes was seen along the epithelial lining (Figure 1). Two of the patients showed the metaplasia of the epithelium (the stratified squamous epithelium instead of the pseudostratified ciliated epithelium). Lamina propria also demonstrated infiltration with lymphocytes, the hypertrophy of glands and the sclerosis of small arterioles (Figure 2). One patient showed the granulation tissue of subepithelium and marked infiltration of lymphocytes, and another patient had the lymphatic nodule of the subepithelium.

The control group patients' nasal mucosa samples where without marked inflammation - no lymphocyte infiltration was seen, seromucosal glands where without hyperplasia.

Numerous PGP-containing nerve fibres $(+++)$ were observed mainly around the secretory parts of seromucosal glands and sclerotic arterioles (Figure 3). The patient with lymphatic nodules in the submucosa demonstred an occasional amount of PGP-containing nerve fibres. The patient who had granulations and the methaplasia of nasal epithelium, 
showed almost negative PGP structures in all the mucosa samples $(0 /+)$ (Table 3).

The control group patients' nasal mucosa samples showed moderate $(++)$ PGP-containing nerve fibres, mostly around blood vessels and seromucosal glands (Table 4).

Nasopharyngeal mucosa showed even more expressed changes in comparison to nasal mucosa - the very thickened basal membrane, the hyperplasia of basal cells, the pronounced hyperplasia of mucosal glands, the sclerosis of small arterioles, some patients had infiltrations of lymphocytes in the submucosa. One patient, the same with granulations in the nasal mucosa, also showed the granulation tissue in the nasopharyngeal submucosa. One of the control group patients had mucoid respiratory epithelium in the nasopharynx, another one had normal respiratory epithelium in the nasopharynx without marked inflammation or hyperplasia.

Table 3. The results of immunohistochemical findings in nasal and nasopharyngeal mucosa

\begin{tabular}{|l|c|c|c|c|c|c|c|}
\hline & PGP & NPY & VIP & SP & CGRP & $\begin{array}{c}\text { Sero- } \\
\text { tonin }\end{array}$ & CgA \\
\hline $\begin{array}{l}\text { Glands - nasal } \\
\text { mucosa }\end{array}$ & $++/++$ & $0 /+$ & ++ & $0 /+$ & $0 /+$ & - & +++ \\
\hline $\begin{array}{l}\text { Glands - } \\
\text { nasopharyngeal } \\
\text { mucosa }\end{array}$ & $++/++$ & + & ++ & $0 /+$ & $0 /+$ & - & +++ \\
\hline $\begin{array}{l}\text { Blood vessels - } \\
\text { Nasal mucosa }\end{array}$ & $++/+++$ & $0 /+$ & ++ & $0 /+$ & $0 /+$ & - & ++ \\
\hline $\begin{array}{l}\text { Blood vessels - } \\
\text { Nasopharyngeal } \\
\text { mucosa }\end{array}$ & +++ & + & ++ & $0 /+$ & $0 /+$ & - & $++/+++$ \\
\hline Total & $++/+++$ & + & ++ & $0 /+$ & $0 /+$ & - & $++/+++$ \\
\hline
\end{tabular}

$(-)$ No positive structure seen in the visual field, $(0 /+)$ rare positive structures seen in the visual field, $(+)$ Occasionally positive structures seen in the visual field, $(+/++)$ few to moderate number of positive structures seen in the visual field, $(++)$ a moderate number of positive structures seen in the visual field, $(++/+++)$ moderate to numerous positive structures seen in the visual field, $(+++)$ numerous positive structures seen in the visual field, $(+++/++++)$ the abundance of positive structures in the visual field. 
Table 4. The results of immunohistochemical findings in nasal and nasopharyngeal mucosa - the control group

\begin{tabular}{|l|c|c|c|c|c|c|c|}
\hline & PGP & NPY & VIP & SP & CGRP & $\begin{array}{l}\text { Sero- } \\
\text { tonin }\end{array}$ & CgA \\
\hline Glands - nasal mucosa & ++ & $0 /+$ & $0 /+$ & $0 /+$ & $0 /+$ & - & + \\
\hline $\begin{array}{l}\text { Glands - } \\
\text { nasopharyngeal mucosa }\end{array}$ & ++ & $0 /+$ & $0 /+$ & $0 /+$ & $0 /+$ & - & + \\
\hline $\begin{array}{l}\text { Blood vessels - } \\
\text { Nasal mucosa }\end{array}$ & ++ & $0 /+$ & $0 /+$ & $0 /+$ & $0 /+$ & - & + \\
\hline $\begin{array}{l}\text { Blood vessels - } \\
\text { Nasopharyngeal mucosa }\end{array}$ & ++ & $0 /+$ & $0 /+$ & $0 /+$ & $0 /+$ & - & + \\
\hline Total & ++ & $0 /+$ & $0 /+$ & $0 /+$ & $0 /+$ & - & + \\
\hline
\end{tabular}

$(-)$ No positive structure seen in the visual field, $(0 /+)$ rare positive structures seen in the visual field, $(+)$ Occasionally positive structures seen in the visual field, $(+/++)$ few to moderate number of positive structures seen in the visual field, $(++)$ a moderate number of positive structures seen in the visual field, $(++/+++)$ moderate to numerous positive structures seen in the visual field, $(+++)$ numerous positive structures seen in the visual field, $(+++/+++)$ the abundance of positive structures in the visual field.

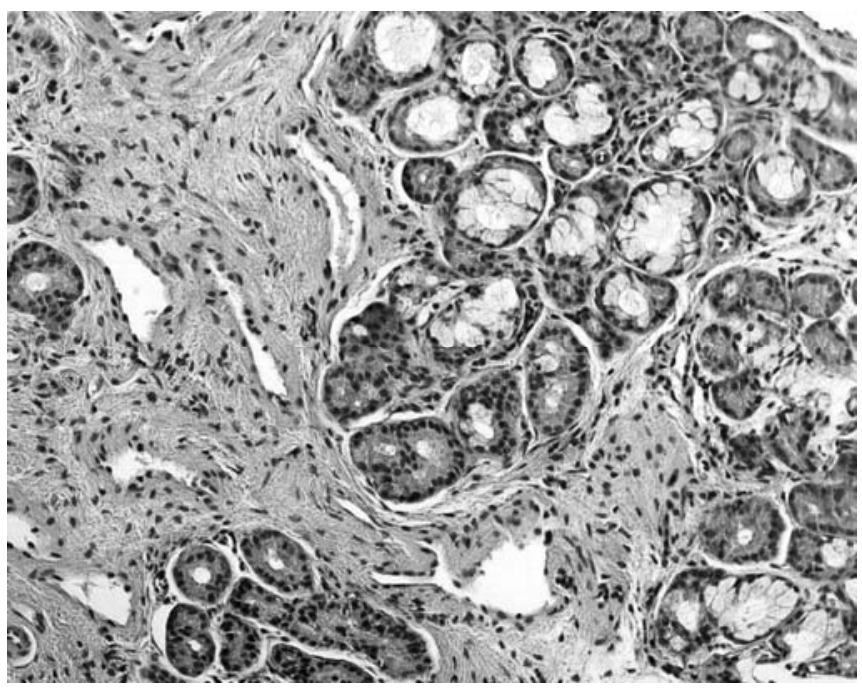

Figure 1. The marked hyperplasia of seromucosal glands, the infiltration of lymphocytes in nasal mucosa, HE x 200. 


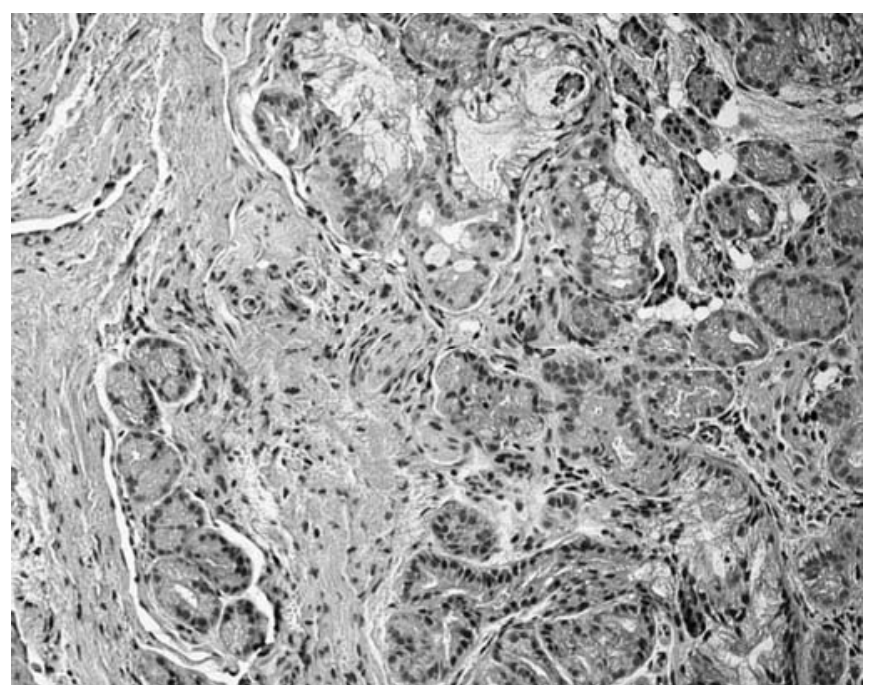

Figure 2. Sclerotic small arterioles, the hyperplasia of seromucosal glands and the infiltration of lymphocytes in nasopharyngeal mucosa. HE x 200.

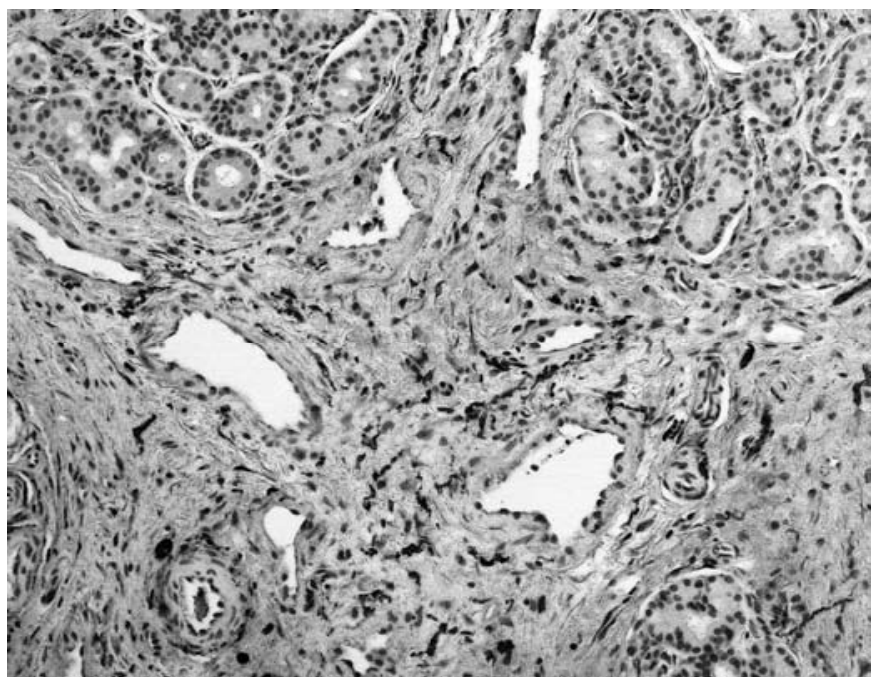

Figure 3. The abundance of PGP immunopositive structures around sclerotic arterioles and hyperplased seromucosal glands in nasopharyngeal mucosa. PGP $9.5 \times 200$. 


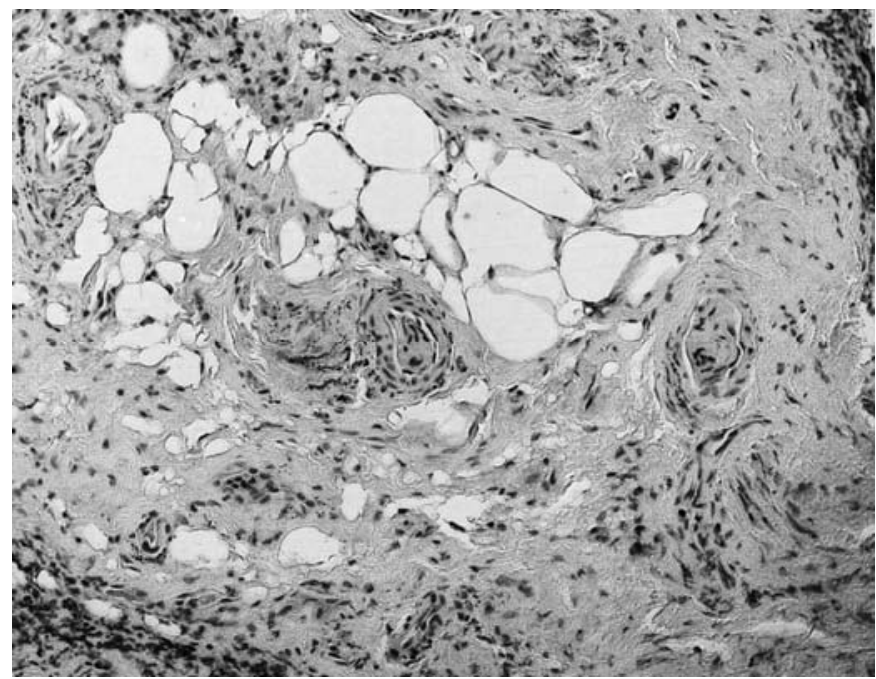

Figure 4. VIP containing nerve fibres around blood vessels in nasopharyngeal mucosa. VIP x200.

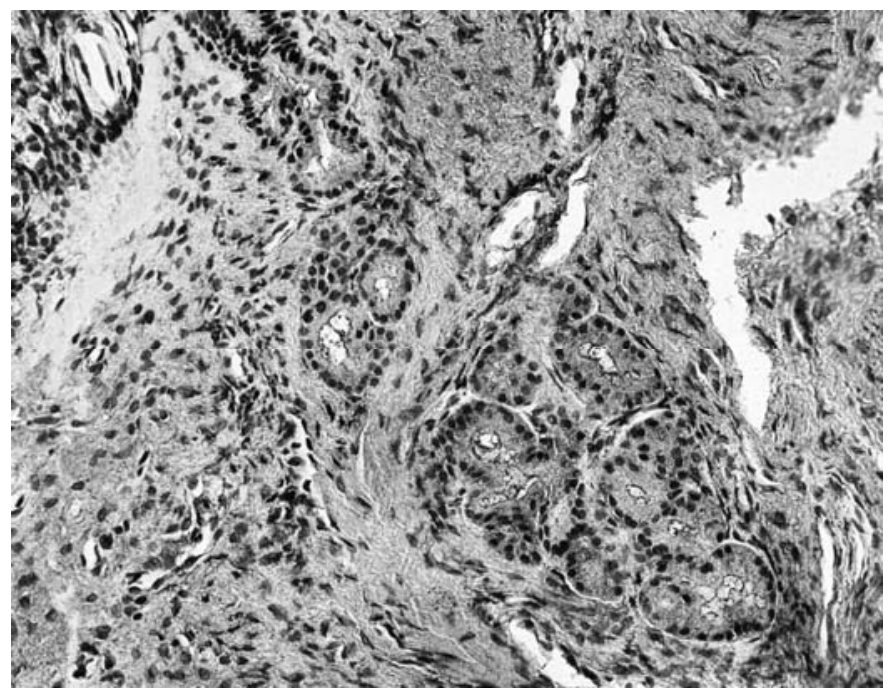

Figure 5. Numerous Chromogranin A granules containing cells next to the hyperplased seromucosal glands. CgA x250. 
The abundance of PGP-containing nerve fibres $(+++/++++)$ was found around glands, sclerotic arterioles in almost all the samples, except the patient with granulations in sub mucosa - he had only few PGPcontaining fibres $(+)$ and only next to some seromucos glands (Figure 4) (Table 3).

The control group patients demonstrated moderate PGP immunopositive structures $(++)$ in nasopharyngeal mucosa, mostly next to seromucosal glands and arterioles.

All the nasal and nasopharyngeal mucosa samples, also in the control group, were serotonin negative (Table 4).

Most of the nasal and nasopharyngeal mucosa samples showed few NPY positive structures (+), mostly around small sclerotic arterioles in the nasopharyngeal mucosa. Exceptions were mucosal samples from the patient with lymphatic nodules in the submucosa, in this case we saw more positive structures in comparison to other patients' mucosa samples around the arterioles in nasopharyngeal mucosa (++) (Table 3$)$.

The control group patients' nasal and nasopharyngeal mucosa showed occasional NPY immunopositive structures $(+)$ next to seromucosal glands and blood vessels (Table 4).

Nasal mucosa showed moderate to a large number of VIP-containing nerve fibres $(++/+++)$ around submucosal glands and small sclerotic arterioles, but in the nasopharyngeal mucosa samples VIP-containing nerve fibres were even more, especially around sclerotic arterioles $(++/+++)$ (Figure 5) (Table 3). The control group patients' nasal and nasopharyngeal mucosa samples showed few VIP immunopositive structures mostly around blood vessels, similarly in nasal and nasopharyngeal mucosa (Table 4).

In all the nasal and nasopharyngeal mucosa samples, including the control group patients, rare $(0 /+)$ or negative Substance $\mathrm{P}$ containing structures were found (Table 3) (Table 4).

Occasional CGRP positive structures $(0 /+)$ in the visual field were seen in the nasal and nasopharyngeal mucosa, mainly in the nasopharyngeal mucosa next to blood vessels and around seromucosal glands, the same findings were seen in the control group patients' mucosa samples (Table 3) (Table 4).

Almost in all of nasal and nasopharyngeal mucosa samples moderate to numerous $(++/+++) \mathrm{CgA}$-containing cells were found, mainly in seromucosal glands and the basal layer of epithelium (Figure 6) (Table 3). Moderate CgA immunopositive structures were found in the 
control group nasal and nasopharyngeal mucosa samples next to blood vessels and glands (Table 4).

\section{DISCUSSION}

This study shows the infiltrations of lymphocytes, thick basal membrane and sclerotic small arterioles and the hyperplasia of seromucosal glands in the nasal and nasopharyngeal mucosa of the patients with the post nasal drip syndrome in contradistinction to the control group patients, whose nasal and nasopharyngeal mucosa samples showed no marked signs of chronic inflammation. Similar results describe also Stephanie and Kunal (2008) by the histological examination of nasal mucosa in the case of allergic rhinosinusitis and hyperreflectoric rhinitis (24).

In the nasopharyngeal mucosa PGP-containing neural fibres were more observed than in the nasal mucosa (in the control group as well as in the post nasal drip patients group), probably due to the density of innervations, which is more pronounced in the mucosa of nasopharynx compared with the mucosa of lower nasal turbinate $(3,7,24)$. PGP imunopositive structures were found less in the control group patients' mucosa samples; probably this finding can be associated with the increased production of neuropeptides in the case of post nasal drip. Fisher \& al (2005) describe similar histological findings and the abundance of PGP-containing nerve fibres in nasal mucosa in the case of persistent perennial allergic rhinitis (9), so we can not exclude some analogy with allergic inflammation in the case of the post nasal drip syndrome pathogenesis.

VIP-containing nerve fibres are usually located around arterioles, glands, and muscle fibres and the most important effects of VIP are vasodilatation, bronchodilatation and the activation of glandular secretion (9). Our study showed moderate to a large number of VIPcontaining nerve fibres around seromucosal glands and small sclerotic arterioles, especially in the nasopharyngeal mucosa samples, it suggests that VIP could implicate in the pathogenesis of mucus discharge in the nasopharynx and probably also stimulate the sclerotisation. Especially according to the fact, that the control group patients' nasal and nasopharyngeal mucosa samples contained a smaller (occasionaly to few) number of VIP imunopositive structures and there were no sclerotic arterioles $(11,12,20)$. 
In our study just rare NPY positive structures were found in nasal and nasopharyngeal mucosa. The control group patients' nasal and nasopharyngeal mucosa samples contained rare NPY immunopositive structures. Heppt \& al (2004) describes similar results in the patients with environmentally triggered hyperreflectory rhinitis (10). Neuropeptide $\mathrm{Y}$ is co-localized with norepinephrine in a population of sympathetic neurons in the walls of human nasal mucosal arterioles (4). Sympathetic stimulation induces vasoconstriction and increased nasal airway potency. There is also some evidence that sympathetic activity can induce airway glandular secretion through the stimulation of serous cells through $\beta$ receptors $(4,19)$. There are studies that reported about the NPY effect on vasoconstriction, it is effective at reducing the symptoms of nasal obstruction and the weight of mucus secretions after the nasal allergen challenge (24). In this case, our findings explain increased mucus formation in the patients' nasopharyngeal mucosa.

In our study we investigated the substance $\mathrm{P}$ appearance in nasal and nasopharyngeal mucosa. The results showed rare or negative $(0 /+) \mathrm{SP}$ containing structures, also in the control group patients' mucosa. Sensory nerve neuropeptides include the tachykinins (i.e., substance P and neurokinin A), the calcitonin gene-related peptide $(4,23)$. The effects of these neuropeptides include glandular activation, leukocyte recruitment, differentiation and the activation of various immune cells, including lymphocytes, eosinophils, mast cells and macrophages (15, $24,25)$. According to these known effects, it is unusual that the substance $\mathrm{P}$ was not found in nasal and nasopharyngeal mucosa samples, and the acquired results may suggest that the substance $\mathrm{P}$ is not so deeply involved in the pathogenesis of the post nasal drip syndrome.

Our results showed the absence of serotonin in all the nasal and nasopharyngeal mucosal samples and also in the control group patients. Serotonin effects are smooth muscle contraction, vasodilatation, and increased vascular permeability. Serotonin as well as histamine, leukotrienes, prostaglandins and tryptase are released and account for the immediate airway allergy symptoms and it is often found in the case of hyperreflectoric rhinitis as well (9). That fact can suggest that serotonin as well as the substance $\mathrm{P}$, has no significant influence on the post nasal drip pathogenesis, and it makes a significant difference from the described study results until now in the case of allergic and hyperreflectory rhinitis $(7,8,9,10,16)$. 
In the studies described in literature, CGRP is usually found in allergic and hyperreflectoric rhinitis $(16,20)$. Calcitonin gene-related peptide is one of the neuropeptides that is released in the human nasal mucosa after trigeminal nerve stimulation. The main effects of this neuropeptide are vasodilatation, mucus secretion, plasma extravasations; CGRP exerted also a significant dose-dependent stimulation on ciliary's beat frequency (4). According to these known effects the negative CGRP-containing nerve fibre in the present study is inexplicable. That seemly suggests the difference between the allergic and the neurogenic inflammation.

Chromogranin A belongs to the granin family of uniquely acidic secretory proteins co-stored and co-secreted with other hormones and peptides in the elements of the diffuse neuroendocrine system. In nasal mucosa chromogranin mediates as a marker of neuropeptides, neuroendocrine cells. CgA peptides take part in the regulation of calcium and glucose metabolism, cardiovascular functions, gastrointestinal motility and nociception, tissue repair, inflammatory responses and as host defence peptides in the first phase of microbial invasions $(4,26,27)$. Our results showed moderate to numerous $\mathrm{CgA}$ immunopositive cells in nasal and nasopharyngeal mucosa in the case of the isolated post nasal drip syndrome. The fact must be mentioned that the control group patients... nasal and nasopharyngeal mucosa samples contained less $\mathrm{CgA}$ immunopositive cells in comparison with the main group patients.

\section{CONCLUSIONS}

The main histological changes in the patients with the isolated post nasal drip syndrome are the thickened basal membrane, the hyperplasia of basal cells, the pronounced hyperplasia of mucosal glands, the sclerosis of small arterioles. The main neuropeptides that are found in nasal and nasopharyngeal mucosa samples are PGP 9.5, the vasoactive intestinal peptide, the neuropeptide $\mathrm{Y}$ and chromogranin in the post nasal drip syndrome group. There is an imbalance of common neuropeptidecontaining innervations, mainly sympathic nerves, the precursors of neuropeptides - chromogranins in the nasopharyngeal mucosa of the patients with the post nasal drip syndrome. SP and CGRP are not the most characteristic neuropeptides in the case of the post nasal drip syndrome pathogenesis. 


\section{REFERENCES}

1. Baraniuk JN. Neural regulation of mucosal function. Pulm Pharmacol Therapy 2008, vol.21: 442-448.

2. Baraniuk J, Saria A, Goff J, Peden D, Merida M, Shelhamer J. Gastrinrelasing peptide tin human nasal mucosa. J Clin Invest 1990, vol.85: 998-1005.

3. Baraniuk J Sensory, parasympathetic, and sympathetic neural influences tin the nasal mucosa. J Allergy Clin Immunol 1992, vol.90: 10451050.

4. Baraniuk J. N, Merck S. J. Neuroregulation of Human Nasal Mucosa. Annals of the newYork Academy of Sciences. 2009, Vol. 170: 604609.

5. Boulais N. PhD, Misery L. MD Merkel cells. Journal of the American Academy of Dermatology 2007, Vol.57:147-165.

6. Dykewicz M S MD, Hamilos D L, MD Rhinitis and sinusitis. Journal of Allergy and Clinical Immunology. 2010, Vol.125:103-115.

7. Fang SY; Shen CL Neuropeptide innervation and neuroendocrine cells in allergic rhinitis and chronic hypertrophic rhinitis. Clin Exp Allergy 1998, vol. 28: 228-32.

8. Fisher A, McGregor GP, Saria A, Philippin B, Kummer W. Induction of tachykinin gene and peptide expression in guinea pig nodose primary afferent neurons by allergic airway inflammation. J Clin Invest 1996, vol. 262:646-653.

9. Fisher A., Wussow A. Cryer A. Neuronal Plasticity in Persistent Perennial Allergic Rhinitis. Journal of Occupational and Environmental Medicine. 2005, vol. 47: 20-25.

10. Heppt W MD, Peiser C MD, Cryer A MD Innervation of Human Nasal Mucosa in Environmentally Triggered Hyperreflectoric Rhinitis. Journal of Occupational and Environmental Medicine 2002, vol. 44:924-929.

11. Heppt W., Thai Dinh Q., Cryer A., Zweng M., Noga O., Peiser C.,. Melvan M, Witt C., Fischer A. and. Groneberg D.A Phenotypic alteration of neuropeptide-containing nerve fibres in seasonal intermittent allergic rhinitis. Clinical \&experimental allergy. 2004, vol. 34: 11051110 .

12. Jeferson C., Dolci J. Neuropeptide immunofluorescence in human nasal mucosa: assessment of the technique for vasoactive intestinal peptide (VIP) Rev. Bras. Otorrinolaringology. 2005, vol.71: 526-534. 
13. Jornot L; Lacroix JS; Rochat T. Neuroendocrine cells of nasal mucosa are a cellular source of brain-derived neurotrophic factor. The European respiratory journal 2008, 32: 769-7401.

14. Jornot L., Grouzmann E., Lacroix J.S., Rochat T. DNF and DPP-IV in polyps and middle turbinates epithelial cells. Rhinology. 2007, vol.45:129-133.

15. Klein Jan A. Basophil and eosinophil accumulation and mast cell degranulation in the nasal mucosa of patients with hay fever after local allergen provocation. J Allergy Clin Immunol. 2000, vol.106: 677-86.

16. Korsgen M, Erjafalt J, Hinterholzl J, Fisher-Colbrie R, Emanuelsson C, Andersson M. Neural expression and increased lavage fluid levels of secretoneurin tin sesonal allergic rhinitis. J Respir Crit Care Med 2003, vol.167:1504-1508.

17. Lacroix J.S. Landis B.N. Neurogenic inflammation of the upper airway mucosa. Rhinology. 2008, vol.46:163-165.

18. Merati A L MD Reflux and cought. Otolaryngologic Clinics of North America. 2010, vol.43:97-110.

19. Mullol J, Rieves RD, Baraniuk JN, Lundgren JD, Hausfeld JH, Shelhamer JH, Kaliner MA. The effects of neuropeptides on mucosus glycoprotein secretion from human nasal mucosa tin vitro. Neuropeptides 1992, vol.21:231-238.

20. Myers A, Kajeka R, Undem B. Allergic inflammation-induced neuropeptide production tin rapidly adapting afferent nerves in guinea pig airways. Am J Physiol Lung Cell Mol Physiol 2002, vol. 282:775781.

21. Ricco MM, Myers AC, Undem BJ. Immunomodulation of afferent neurons in guinea pig isolated airway. J Physiol 1996, vol.491:499509.

22. Salib R. J., Harries P. G., Nair S. B, Howarth P. H Mechanisms and mediators of nasal symptoms in non-allergic rhinitis. Clinical \& Experimental Allergy. 2008, vol. 38:393-404.

23. Sanu A, Eccels R. Postnasal drip syndrome. Two hundred years of controversy between UK and USA. Rhinology. 2008, vol. 46: 86-91.

24. Seema S MD, Undem B, PhD, Alvin S, MD, Togias A, MD The role of the nervous system in rhinitis Journal of Allergy and Clinical Immunology. 2006, vol. 118:

25. Stephanie A.J., Kunal T. Chronic rhinosinusitis and Asthma. Otolaryngologic Clinics of North America 2008, vol. 41:297-309.

26. Tai C, Baraniuk J. Upper airway neurogenic mechanisms. Curr Opin Allergy Clin Immunol 2002, vol. 2:11-9. 
Address for correspondence

Gunta Sumeraga

Department of Otorhinolaryngology

Pauls Stradins Clinical University Hospital

Pilsonu 13, Riga, Latvia, LV-1002

E-mail: gunta_sumeraga@inbox.lv 